\title{
The Journal of
}

COLLEGIATE EMERGENCY MEDICAL SERVICES

ISSN: 2576-3687 (Print) 2576-3695 (Online) Journal Website: https://www.collegeems.com

\section{From Volunteer Student Organization to Official Division of a University Department}

Tracing the History of Arizona State University Emergency Medical Services Jada Wang

Keywords: collegiate-based emergency medical services, finance, management

Citation (AMA Style): Wang J. From Volunteer Student Organization to Official Division of a University Department: Tracing the History of Arizona State University Emergency Medical Services. J Coll Emerg Med Serv. 2020; 3(1): 11-15. https://doi.org/10.30542/ JCEMS.2020.03.01.04

Electronic Link: https://doi.org/10.30542/JCEMS.2020.03.01.04

Published Online: February 28, 2020

Published in Print: February 28, 2020 (Volume 3: Issue 1)

Copyright: (C) 2020 Wang. This is an OPEN ACCESS article distributed under the terms of the Creative Commons Attribution 4.0 International (CC BY 4.0) License, which permits unrestricted use, distribution, and reproduction in any medium, provided the original author and source are credited. The full license is available at: https://creativecommons.org/licenses/by/4.0/ 


\section{From Volunteer Student Organization to Official Division of a University Department: \\ Tracing the History of Arizona State University Emergency Medical Services}

Jada Wang, NRP, BS, MS

ABSTRACT

Background: Student Emergency Medical Services (SEMS) at Arizona State University (ASU) began as a student-run volunteer organization in 2008. SEMS provided two types of EMS services: 911 Response and Special Event Standby, staffed by volunteer EMTs and volunteer dispatchers. Case Report: In 2016, SEMS leaders formally proposed for SEMS to become a university department under the name ASU EMS. In 2018, ASU EMS became an official division of the ASU Fire Marshal's Office within the ASU Environmental Health and Safety (EHS) Department. SEMS with student volunteers transformed to ASU EMS with paid student workers. This case report traces the history of ASU EMS from 2008 to 2019. Discussion: The transition process from a student volunteer group to an official division of the university with paid workers was time and work intensive. However, the transition ultimately afforded

KEYWORDS: collegiate-based emergency medical services; finance; management

Corresponding Author and Author Affiliations: Listed at the end of this article. numerous benefits to the organization. As opposed to SEMS, ASU EMS has had an easier hiring process, a higher retention rate for student EMS providers, and a consistent $100 \%$ staffing level for shifts. Within the first two semesters of its transition, ASU EMS employed 28 student workers, trained approximately 800 people in CPR, and experienced a 122\% increase in on-campus EMS standby requests and a $128 \%$ increase in gross revenue. Conclusions: Other collegiate EMS leaders and advisors may consider the lessons learned for the development of their collegiate EMS organizations.

$\mathbf{S}$ tudent Emergency Medical Services (SEMS) was founded as an all-student-run volunteer organization at Arizona State University (ASU) in 2008, funded by the undergraduate student government. At that time, the university had over 67,000 enrolled students and four campuses. ${ }^{1}$ Over the next 10 years, SEMS evolved into ASU EMS, an official division of a university department with paid positions for one administrative staff and over 20 student workers. ASU now has a total of 72,709 enrolled students spread among five campuses, located in the cities of Tempe, Phoenix, Glendale, Mesa, and Lake Havasu. ASU EMS operates on all five campuses for Special Event Standby coverage, and operates only on the main campus at Tempe for 911 Response coverage. The Tempe Fire Department (Tempe FD) is required by law to respond to all 911 calls on campus. However, during 911 Response shifts, an ASU EMS team arrives on scene first and initiates care. In instances of low-acuity patients or patients who refuse transport, the Tempe FD often allows ASU EMS to assume full responsibility of the patient and finish providing care on scene. Occasionally, ASU EMS

\footnotetext{
Jada Wang, NRP, BS, MS obtained her Bachelor (2018) and Master (2019) of Science in Biology at Arizona State University (ASU). She joined ASU Emergency Medical Services in 2014 after qualifying as an EMT. Later, as a paramedic, Jada served as the organization's EMS Coordinator. She also completed a vehicular internship with Phoenix (AZ) Fire Department and worked as a paramedic in nearby Scottsdale. She is now an adjunct faculty member for Maricopa Community Colleges.
}

then provides courtesy rides for patients to their dorm rooms, ASU Health Services, or other destinations on campus. ASU EMS is regulated by Arizona Department of Health Services (AZDHS), and all EMT providers are AZDHS-licensed. ASU EMS teams consist of at minimum 2 EMT members, and they carry a full set of equipment as defined by AZDHS. Teams are integrated into the campus 911 dispatch system. ASU EMS team members receive regular continuing education trainings, and all operate under a medical director who is a board-certified emergency medicine physician. ASU EMS is a service that is tailored to the needs of the university and its populations.

This case report details the organization's evolution from a volunteer group to an official division of a university department. Other collegiate EMS leaders and advisors may consider this case report as an example for future desired transitions and development of their collegiate EMS organizations. To understand the history and development of ASU EMS since its establishment in 2008, interviews were conducted with key stakeholders. Subjects included administrative staff who partook in the process of helping SEMS to transition to ASU EMS. In addition to in-person interviews, detailed tracking of internal documents saved in a shared Google Drive folder for SEMS leadership was also completed. The SEMS Google Drive includes documents that cover the entire time range in question, from 2008 to the summer semester of 2018. 


\section{Case Report}

\section{Establishment of Student EMS}

SEMS was originally established to unite young students who were interested in serving their community by providing prehospital emergency care. The process of establishing SEMS was fairly easy to facilitate, as it was categorized as a student organization. The only requirements in this process were to recruit volunteer members and to find an advisor for the organization. Dr. Stefanie Schroeder, a board-certified emergency medicine physician and Chief of Medical Staff at ASU Health Services, agreed to serve as the Medical Director and official advisor to SEMS. She has remained a supporter and promoter for the organization ever since, and she is the Medical Director for ASU EMS to this day.

When SEMS founders first sought to establish and structure the
This was due primarily to Dr. Schroeder's dual roles as Medical Director for SEMS and Chief of Medical Staff at ASU Health Services. For example, Dr. Schroeder played a critical role in drafting and approving medical protocols for SEMS based on national EMS standards. Additionally, Dr. Schroeder facilitated the establishment of SEMS' jurisdiction. In Arizona, EMS systems are fire-based, and the main campus at Tempe was under the jurisdiction of the Tempe Fire Department (Tempe FD). Therefore, conflicts emerged when firefighters expressed discomfort with college-aged EMTs responding to 911 calls. Dr. Schroeder advocated for SEMS in the face of this pushback and initiated meetings between Tempe FD chiefs and SEMS leaders to resolve these conflicts.

\section{Financial Sustainability}

SEMS received minimal pushback from the university due to its

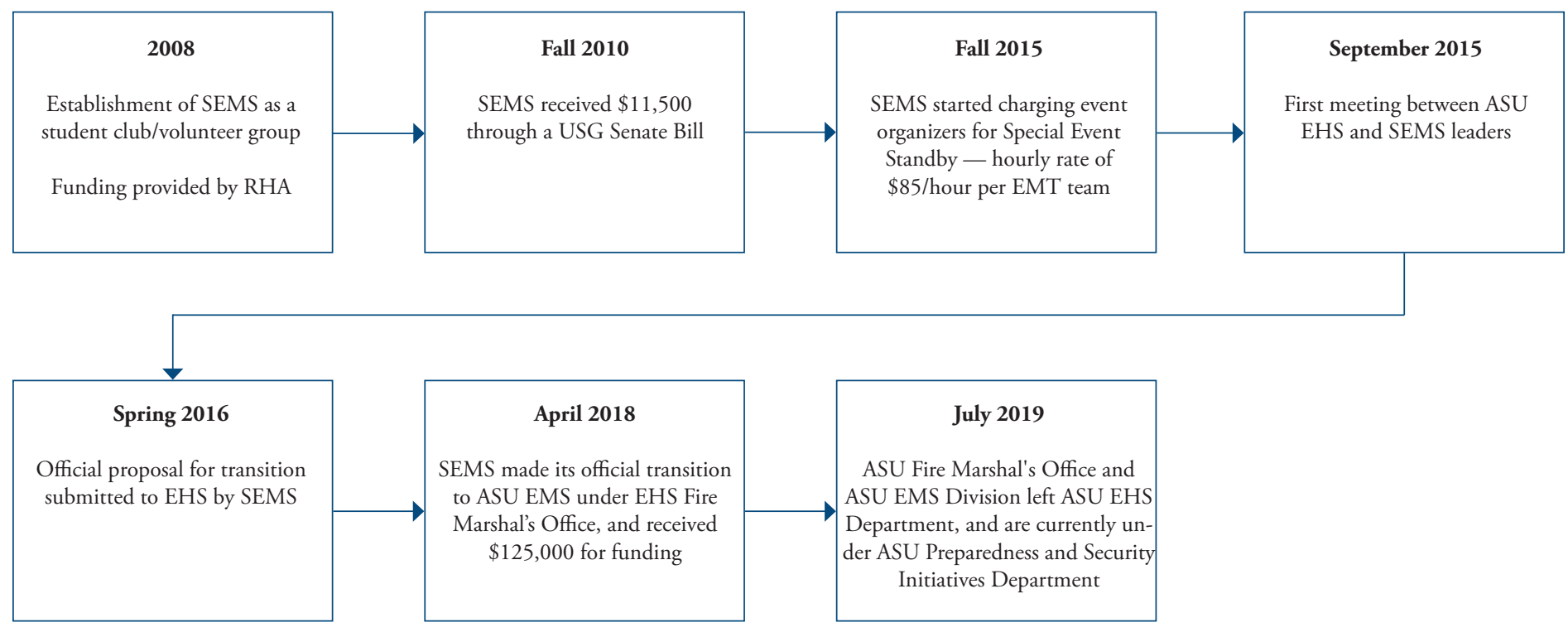

Figure 1. Important milestones in the transition process from Student EMS (SEMS) to ASU EMS.

organization in 2008, the ASU administration offered the founders the opportunity to become an official division under their Health Services department (Figure 1). However, if SEMS were taken under ASU Health Services, their EMTs and paramedics would have had a severely limited scope of practice due to liability concerns. ASU Health Services expressed a belief that SEMS was at higher risk of malpractice due to the young age and lack of experience of collegiate EMTs. This limited scope of practice would not have included the administration of medications such as oxygen or oral glucose, and would have afforded SEMS providers limited authority to make independent medical decisions. To avoid limiting the potential of the organization, the founders opted instead to establish themselves as a volunteer group outside of any university department. As an independent group of volunteers, SEMS members were able to utilize their full scope of practice.

Although SEMS did not become an official division of ASU Health Services, the two organizations maintained a close relationship and SEMS received considerable guidance and support from the Health Services department during early phases of development. status as a student organization, and because many event organizers on campus appreciated having EMS services on standby. SEMS was able to receive funding as a student organization and a 501(c) (3) non-profit organization. Initial funding for SEMS was provided entirely by the ASU Residence Hall Association (RHA). Within one year of its inception, SEMS began receiving some money from the ASU Undergraduate Student Government (USG) Appropriation process. In Fall 2010, SEMS received \$11,500 through a USG Senate Bill (Figure 1). RHA started slowly decreasing financial support for SEMS in Fall 2010 and discontinued all support in 2012. USG funding for SEMS never exceeded over $\$ 10,000$ per year, and typically remained between $\$ 5,000$ to $\$ 8,500$. SEMS also received donations from the Panhellenic Council and ASU Sun Devil Athletics. SEMS originally provided EMS coverage for special events on ASU campuses free of charge. However, to make the organization more financially self-sustaining, SEMS leadership decided in Fall 2015 to charge an hourly rate for special event coverage of $\$ 85 /$ hour per team consisting of 2 EMTs (Figure 1). ASU EMS office space located in McClintock Residence Hall is still provided free of charge 


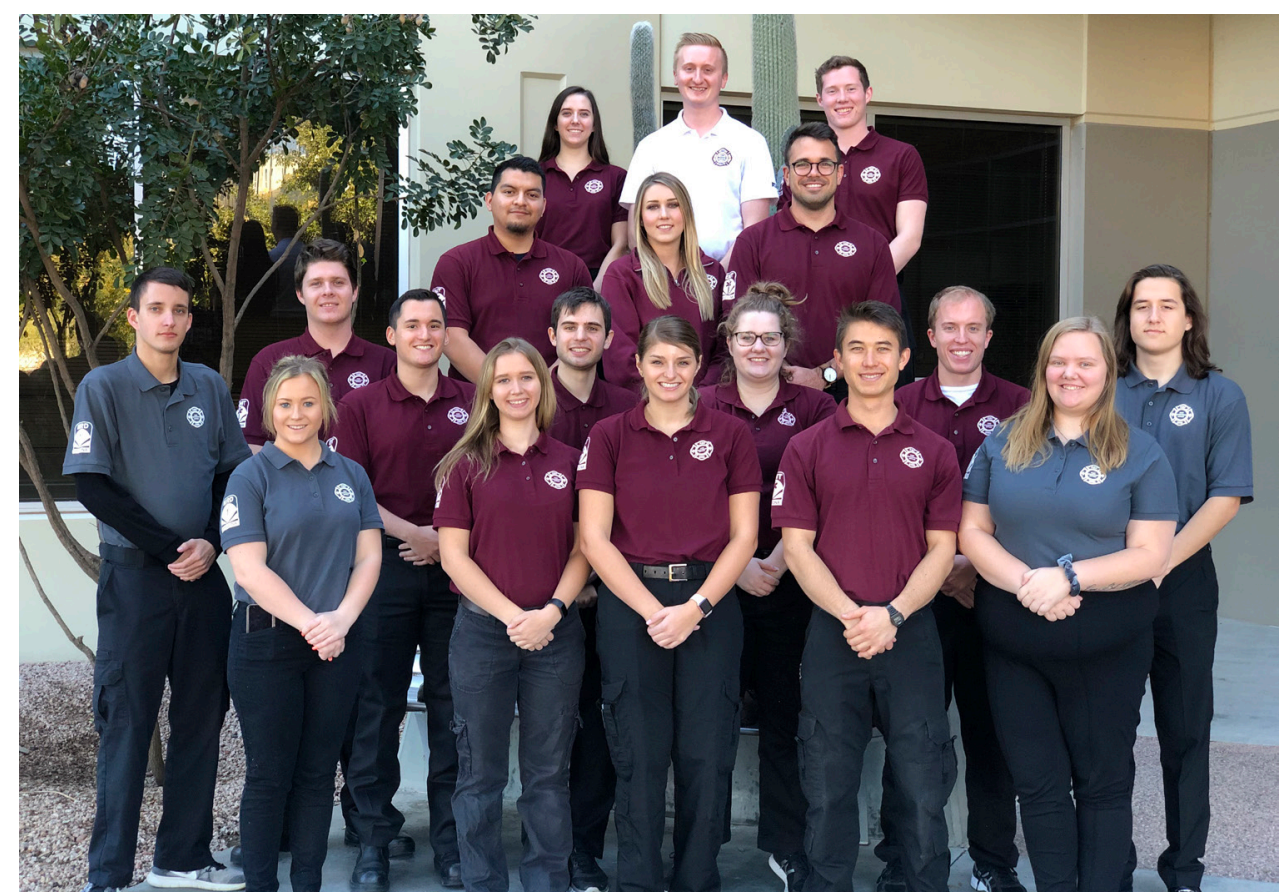

ASU EMS team during Fall 2019 pre-semester training. This is the first group of student workers hired by ASU EMS after its official transition. standby requests. Many special events prior to the year 2015 were handled through email, phone calls, or face-to-face interactions, and were not logged. However, SEMS was ultimately successful in making the case that SEMS' services were needed on campus, and that demand was increasing over time.

During these early meetings, the vision of the main stakeholders was to restructure SEMS as a professional service center similar to ASU Health Services, under the name ASU EMS with volunteer EMTs and dispatchers as well as a part-time paid administrator. The stakeholders anticipated that it would be easier for SEMS to transition if there was only one paid staff position, since a smaller budget would be required from the university. All stakeholders agreed that a primary goal in this transition was for ASU EMS to eventually be self-funded. Therefore, stakeholders decided to keep constant the original rate of $\$ 85 /$ hour per team for Special Event Standby requests so as to maintain the network SEMS had formed with event organizers and other university departments. Additionally, the ASU Fire Marshal proposed a policy which would limit ASU organizers' ability to hire outside EMS/first aid groups. In theory, this policy would encourage utilization of ASU EMS services on campus, increasing gross revenues. In addition, this policy would reduce costs for the university, as SEMS event standby coverage rates for a two-member team were substantially lower than local competitors (Table 1).

\section{Proposal Development and Iterations}

Over the course of several months, stakeholders continued to develop their vision for ASU EMS. By the time the final proposal was submitted to ASU, three large changes had been made. First, the paid administrative staff position would be full-time, not parttime, under the title of EMS Coordinator. The EMS Coordinator would oversee personnel, finance, logistics, operations, and outreach for ASU EMS. SEMS leaders and EHS agreed that a full-time staff member was needed to initiate and lead a newly formed university EMS organization. Second, student EMS providers would be paid workers as opposed to volunteers. Student workers would receive an hourly wage determined by their ranking in the organization. SEMS leaders and EHS believed this would increase retention of student workers as well as the organization's capacity to staff 911 Response and Special Event Standby shifts. Finally, the proposal did not include a policy limiting on-campus hiring of off-campus EMS/ first aid group as stakeholders agreed that it would not be feasible for ASU EHS to implement.

In early 2016, the Business Operations Manager at EHS and ASU's Director of Business Applications and Fiscal Control met with ASU Financial Services and passed along the final written proposal (Figure 1). This proposal justified the need for initial funding from ASU, explained why a paid administrator was essential for the success of the organization, and demonstrated how ASU EMS would 
Table 1. Examples of Arizona EMS agencies and associated costs for event standby coverages with two providers. ${ }^{2}$

\begin{tabular}{lcccc}
\hline Agency Name & Type of Agency & Type of Entity & Staffing Level & Standby Rate \\
\hline ABC Ambulance & Independent & For Profit & 1 Paramedic, 1 EMT & $\$ 158.65 / \mathrm{hr}$ \\
$\begin{array}{l}\text { American Medical Response of } \\
\text { Maricopa }\end{array}$ & Independent & For Profit & 1 Paramedic, 1 EMT & $\$ 206.70 / \mathrm{hr}$ \\
LifeLine Ambulance Service & Independent & For Profit & 1 Paramedic, 1 EMT & $\$ 163.20 / \mathrm{hr}$ \\
Ajo Ambulance & Independent & Nonprofit & 1 Paramedic, 1 EMT & $\$ 433.47 / \mathrm{hr}$ \\
Tempe Fire Department & Fire Department & Municipality & 1 Paramedic, 1 EMT & $\$ 206.70 / \mathrm{hr}$ \\
\hline
\end{tabular}

eventually become self-funded. To effectively make this case, SEMS collected data on its operations and finances and made predictions for volunteer hours and revenue return for subsequent fiscal years.

The proposal for the official transition from SEMS to ASU EMS took almost 3 years to move through the necessary process (Figure 1). During this wait period, there was limited information provided to SEMS leaders from the university. SEMS leaders regularly reached out to EHS asking for updates and were instructed to keep waiting throughout the transition period. Ultimately, however, the proposal was approved with minimal modifications.

\section{Official Transition to University Department}

On April 1, 2018, SEMS officially made its transition to ASU EMS under the EHS Fire Marshal's Office and received \$125,000 of funding from the office of the Chief Financial Officer for the fiscal year of 2018-19 (Figure 1). This budget was intended to hire an EMS Coordinator and several student worker staff as well as to purchase necessary equipment and supplies. Soon after the transition, the EMS Coordinator and ASU Assistant Fire Marshal conducted interviews to hire student EMS workers. The majority of the initial applicants had previously volunteered with SEMS. Prior to the transition, SEMS had over 40 volunteer EMTs and dispatchers. Post-transition, ASU EMS maintained a size of approximately 20 student workers, although this size has fluctuated depending on student schedules and school workload. In total, 28 student workers have been employed since the transition. The process of hiring student workers was found to be easier than recruiting student volunteers. Additionally, the post-transition retention rate has been higher and student workers have tended to work more shifts than volunteers. Therefore, despite a decrease in the overall roster of the organization, ASU EMS has managed to staff Special Event Standby and 911 Response shifts at higher levels and more consistently.

In July 2019, the Fire Marshal's Office and ASU EMS left the EHS Department and relocated under the Preparedness and Security Initiatives Department. This transfer has not notably impacted ASU EMS, as it remains a division of the Fire Marshal's Office and all operations remain the same.

\section{Discussion}

The transition from SEMS to ASU EMS was long, hard, complicated, and required many individuals lending support. The process was particularly stressful and demoralizing for student EMS leaders, who underwent years of effort to achieve a result that, for many, did not arrive during their time on campus. Additionally, throughout the transition period, many major decisions were out of the student leaders' hands, and they were provided limited updates and information. Despite these barriers, the success of this process has led to numerous benefits for the organization. By housing ASU EMS within an official university department and compensating student EMS providers, the organization has increased the quantity of its services, diversified its role on campus, and promoted its own financial wellbeing.

Prior to the transition, SEMS leaders occasionally canceled 911 Response shifts or denied requests for EMS standby at events. This was primarily due to staffing shortages as a result of conflicts with members' school and work schedules. Now that ASU EMS compensates its student workers with an hourly wage, many student EMS providers rely on this income in place of other jobs. Fewer work-related schedule conflicts have largely eliminated staffing shortages for ASU EMS. As a result, the organization has expanded its capacity to consistently staff 911 response shifts on Friday and Saturday nights, and has maintained 100\% staffing level for all 911 Response and Special Event Standby shifts.

Since the transition, ASU EMS has received a steadily increasing number of Special Event Standby requests. While ASU EMS is currently unable to release specific financial information such as gross revenue or wages, the organization continues to receive an annual budget from the university for each fiscal year. The original vision for ASU EMS to be self-funded remains unchanged. ASU EMS aims to eventually generate more revenue through special event coverage than their annual budget, in which case it would no longer require university funding.

Being an official university organization has allowed ASU EMS to expand its functions due to increased funding levels and advocacy on its behalf from a parent department. For example, ASU EMS is now tasked with routinely inspecting automated external debrillators (AEDs) on all ASU campuses. Additionally, ASU EMS has implemented a series of classes to the ASU community on CPR, bleeding control, and opioid awareness and response. Since the transition, approximately 800 people have been trained in CPR on campus. By offering these classes, ASU EMS generates additional revenue, promotes overall campus health, and advertises ASU EMS to the campus community. 
Prior to the transition, SEMS received occasional pushback or disapproval from the Tempe FD due to concerns about student-aged EMTs responding to 911 medical calls. Legitimizing ASU EMS as an official division within the university has strengthened the relationship between student EMTs and the Tempe FD. Since the transition, the EMS Coordinator has started meeting regularly with Tempe FD Deputy Chiefs and Captains. EMT supervisors have also pushed for more ride-alongs and visits to the FD stations. Due to a resulting higher level of comfort and familiarity with student EMTs, as well as more consistent staffing levels, ASU EMS has increased its 911 call volume with Tempe FD. The Tempe FD has started seeing ASU EMS as an asset instead of a liability.

For students at universities who are considering starting a collegiate-based EMS (CBEMS) organization, or for existing volunteer-based CBEMS organizations considering transitioning to an official division within a university department, it is important to remember to be persistent, patient, and determined. When considering becoming an official division, office, or department, organizations should carefully think about to which university 'parent' department the CBEMS organization would best belong. Organizations should then reach out to the department leaders and present why the proposed change would benefit the department, university, and larger campus community. In order to make this case effectively, CBEMS organizations should gather data that demonstrate the need for EMS coverage on campus, such as number of students, staff, and daily visitors, and number of EMS calls on campus. It is also important to demonstrate how establishing an EMS program on campus would be financially feasible for the university and how revenue would be generated. If possible, showing how a CBEMS organization could alleviate on-campus call volumes from local fire or EMS departments would also be beneficial. Beyond demonstrating need, critical to this process is finding a department leader or advisor who believes in the mission of the CBEMS organization and is willing to be an advocate for the organization. Receiving funding from the university financial office is especially challenging in the absence of an administrative advocate for the organization.

\section{Conclusions}

From a student organization to an official division of a university department, the transition process for ASU EMS was time-consuming. However, ASU EMS finally received the budget to be funded as an official division under a university department in 2018. ASU EMS now works alongside the ASU Fire Marshal's Office, the Preparedness and Security Initiatives Department, ASU Health Services, and the Tempe Fire Department. ASU EMS continues to have a steady increase in requests for EMS standby coverage and other campus services such as AED inspections and CPR classes. In place of other part-time employment, student EMTs can work for the ASU community doing something they love while putting their skills, certifications, and training to use. Since the transition, there has been a constant 100\% staffing level for 911 Response and Special Events Standby shifts. ASU EMS has also seen an increase in gross revenue, with the ultimate goal to be self-funded. By working shifts and offering courses across campus, the organization is promoting the ASU Fire Marshal's Office and the EMS program to the ASU community. There are also better working relationships among ASU departments with better coordination. Ultimately, despite a long and laborious process, ASU EMS has transformed for the better.

\section{Acknowledgments}

I would like to acknowledge my thesis advisors from Arizona State University (ASU) - Dr. Matt Chew, Dr. Jane Maienschein, Dr. Karin Ellison, and Dr. Alison Essary.

I thank them for their assistance with my research and writing, and their unfailing support and encouragements. I would like to acknowledge the Center for Biology and Society at ASU, and the ASU Fire Marshal's Office.

\section{References}

1. Arizona State University. Enrollment Trends - Metropolitan Campuses. https://facts.asu.edu/Pages/Enrollments/Enrollment Trends.aspx. Accessed December 2019.

2. Arizona Department of Health Services, Bureau of Emergency Medical Services and Trauma System. Arizona Ground Ambulance Service Rate Schedule. https://www.azdhs.gov/documents/preparedness/emergencymedical-services-trauma-system/ambulance/ground/rates/2018/groundambulance-rates-jan-2018.pdf. Published January 8, 2018. Accessed December 2019.

Author Affiliations: From Center for Biology \& Society, Arizona State University School of Life Sciences and Emergency Medical Services, Arizona State University Fire Marshal's Office - both in Tempe, AZ, USA (J.W.)

Address for Correspondence: Jada Wang, NRP, BS, MS.

E-mail: Jada.Wang@asu.edu

Conflicts of Interest/Funding Sources: By the JCEMS Submission Declaration Form, all authors are required to disclose all potential conflicts of interest and funding sources. All authors declared that they have no conflicts of interest. All authors declared that they did not receive funding to conduct the research and/or writing associated with this work.

Authorship Criteria: By the JCEMS Submission Declaration Form, all authors are required to attest to meeting the four ICMJE.org authorship criteria: (1) Substantial contributions to the conception or design of the work; or the acquisition, analysis, or interpretation of data for the work; AND (2) Drafting the work or revising it critically for important intellectual content; AND (3) Final approval of the version to be published; AND (4) Agreement to be accountable for all aspects of the work in ensuring that questions related to the accuracy or integrity of any part of the work are appropriately investigated and resolved.

Prior Publication: Information from this case report was included in an unpublished Master's Thesis: Wang J. Investigating the Current Status of Collegiate Emergency Medical Services (EMS) Organizations via a National Survey Study and Tracing the History of Arizona State University EMS Department [Unpublished master thesis]. Tempe, AZ: Arizona State University. 2019.

Submission History: Received December 27, 2019; accepted for publication February 19, 2020.

Published Online: February 28, 2020

Published in Print: February 28, 2020 (Volume 3: Issue 1)

Reviewer Information: In accordance with JCEMS editorial policy, Case Report manuscripts undergo double-blind peer-review by at least two independent reviewers. JCEMS thanks the anonymous reviewers who contributed to the review of this work.

Copyright: () 2020 Wang. This is an open access article distributed under the terms of the Creative Commons Attribution 4.0 International (CC BY 4.0) License, which permits unrestricted use, distribution, and reproduction in any medium, provided the original author and source are credited. The full license is available at: https:// creativecommons.org/licenses/by/4.0/

Electronic Link: https://doi.org/10.30542/JCEMS.2020.03.01.04 